Original article

\title{
Stress and cognitive biases in schizotypy: A two-site study of bias against disconfirmatory evidence and jumping to conclusions
}

\author{
Thanh P. Le ${ }^{a, *}$, Taylor L. Fedechko ${ }^{a}$, Alex S. Cohen $^{a}$, Samantha Allred ${ }^{a}$, Carrie Pham $^{a}$, \\ Shôn Lewis ${ }^{b}$, Emma Barkus ${ }^{c}$ \\ a Department of Psychology, Louisiana State University, United States \\ ${ }^{\mathrm{b}}$ Division of Psychology and Mental Health, University of Manchester, United Kingdom \\ ' School of Psychology, University of Wollongong, Australia
}

\section{A R T I C L E I N F O}

\section{Article history:}

Received 10 May 2019

Received in revised form 17 August 2019

Accepted 26 August 2019

Available online 8 September 2019

\section{Keywords:}

Schizotypy

Reasoning bias

Cognition

Schizophrenia

Positive

Cognitive bias

\begin{abstract}
A B S T R A C T
The dysfunctional cognitive and reasoning biases which underpin psychotic symptoms are likely to present prior to the onset of a diagnosable disorder and should therefore be detectable along the psychosis continuum in individuals with schizotypal traits. Two reasoning biases, Bias Against Disconfirmatory Evidence (BADE) and Jumping to Conclusions (JTC), describe how information is selected and weighed under conditions of uncertainty during decision making. It is likely that states such as elevated stress exacerbates JTC and BADE in individuals with high schizotypal traits vulnerable to displaying these information gathering styles. Therefore, we evaluated whether stress and schizotypy interacted to predict these reasoning biases using separate samples from the US (JTC) and England (BADE). Generally speaking, schizotypal traits and stress were not independently associated with dysfunctional reasoning biases. However, across both studies, the interaction between schizotypy traits and stress significantly predicted reasoning biases such that increased stress was associated with increased reasoning biases, but only for individuals low in schizotypal traits. These patterns were observed for positive schizotypal traits (in both samples), for negative traits (in the England sample only), but not for disorganization traits. For both samples, our findings suggest that the presence of states such as stress is associated with, though not necessarily dysfunctional, reasoning biases in individuals with low schizotypy. These reasoning biases seemed, in some ways, relatively immutable to stress in individuals endorsing high levels of positive schizotypal traits.
\end{abstract}

(c) 2019 Elsevier Masson SAS. All rights reserved.

\section{Introduction}

Schizophrenia is among the costliest human conditions [1,2]. Identifying individuals at-elevated risk for developing schizophrenia-spectrum disorders is critical to understanding these disorders, improving outcomes, and decreasing their societal burden [3]. Schizophrenia is largely considered an extreme variant of a genetically-mediated constellation of traits, what many refer to as schizotypy [4]. Schizotypy has been defined as continuum of traits associated with schizophrenia-spectrum pathology [4,5]. Moreover, it is a unifying construct that encompasses several different phenotypes for psychosis risk including: biological family members of individuals with schizophrenia, adolescents and early adults with various

\footnotetext{
* Corresponding author at: Department of Psychology, Louisiana State University, 236 Audubon Hall, Baton Rouge, LA 70808, United States.

E-mail address: tle87@lsu.edu (T.P. Le).
}

psychiatric presentations as assessed by clinical interviews such as the Structured Interview of Psychosis-risk Syndromes (e.g., high risk studies), and individuals with elevated self-reported (i.e., "psychometric") schizotypal traits. The latter approach represents the majority of schizotypy research to date and is the focus of the present studies. As schizotypy is associated with higher risk for developing schizophrenia-spectrum pathology, this personality organization has become the focus of early identification and intervention attempts [6,7].

Dysfunctional cognitive and reasoning biases are among the most studied vulnerability markers associated with schizophreniaspectrum disorders and schizotypy [8-10]. While cognitive and reasoning biases may be helpful and even adaptive in some contexts (i.e., increased efficiency and decision making), these biases can also lead to misguided and incorrect judgements - for example, turning benign social interactions into potentially suspicious encounters. These more dysfunctional biases are prominent schizophrenia-spectrum disorders and associated with clinical states such as delusions and hallucinations [11] and social 
dysfunction [12]. Specifically, the Bias Against Disconfirmatory Evidence (BADE) and Jumping To Conclusions (JTC) are two theoretically and empirically linked [11] dysfunctional reasoning biases related to the emergence, expression, and exacerbation of schizotypal traits and clinically diagnosed psychosis $[13,14]$. BADE is characterized by a thinking style easily dismissive of evidence contradicting a held belief and JTC is defined as strong conviction in an idea prior to gathering much or any evidence [13]. These dysfunctional cognitive biases are thought to be the underlying mechanism for paranoia and suspiciousness for individuals on the schizophrenia-spectrum [12,15]. As noted earlier, this style of thinking is also related to neuropsychological and social functioning $[16,17]$. Given their links to functioning, these maladaptive biases are major focal points in interventions (e.g., Metacognitive Training, Social Cognition Interaction Training) that aim to enhance overall and social functioning [17,18].

There are several important knowledge gaps regarding dysfunctional reasoning biases (i.e. BADE and JTC) in individuals with schizotypy. First, considerable variability exists in the strength of these biases measured in different samples across studies [19]. Some factors explaining this variability have been identified including difficulty of stimulus detection, also known as perceptual load, of the task [20]; state anxiety [21]; and the ability to detect incorrectness and change answers, also known as feedback sensitivity [13]. Collectively, these results suggest that reasoning biases may be dynamic as a function of stimulus features, state emotion, and cognitive flexibility. Related to these potential mechanisms, a promising and unexplored moderating variable in the relationship between schizotypy symptoms and these cognitive biases is perceived psychological stress. Cohen and colleagues [22] defined perceived psychological stress as varying degrees of global appraisals regarding which situation in one's life are stressful. This type of stress is similar to state anxiety, or the momentary worry for a threatening demand, yet is much more inclusive in that perceived psychological stress encompasses subjective reactions or appraisals (e.g., unpredictable, uncontrollable, overloaded) to previous, immediate, and upcoming life events or threats. Thus, it stands to reason that stress may exacerbate reasoning biases. Indeed, Keefe and colleagues [23] observed increased reasoning biases following experimentally induced stress in delusion-prone university students, though there are conflicting findings (see [24]). Furthermore, schizotypy is associated with extreme subjective experience of cognitive and emotion regulation deficits, even though studies that use objective measures often fail to observe deficits that approach these elevated subjective levels $[25,26]$. Thus, measuring perceived stress could be an important key to understanding the potential mechanism of reasoning biases in this population.

A related gap in the literature is the relationship between dysfunctional reasoning biases (i.e., BADE and JTC) and the differing trait clusters of schizotypy (i.e., positive, negative, and disorganized; [27]) The vast majority of studies to date examining the relationship between reasoning biases and schizotypy have almost exclusively examined positive schizotypy traits, or related phenomenon such as delusion proneness [14,15]. This is likely due to a generally accepted theoretical mechanism that dysfunctional reasoning biases underlie positive symptoms [11,14,15]. Moreover, positive symptoms (i.e., clinically significant delusions) and subclinical related experiences such as aberrant salience and suspiciousness are linked with prefrontal-limbic connections and dopaminergic functioning, each themselves sensitive to stress $[28,29]$. As such, there is evidence generally suggesting a significant relationship between positive schizotypal traits and reasoning biases $[14,20]$. Even so, not all studies have consistently found that relationship to be significant [30], thus necessitating further research. Moreover, not much evidence has been gathered to document the relationship between negative or disorganized traits of schizotypy and reasoning biases [19]. Schizotypy is heterogeneous construct and therefore associated with a wide range of symptoms. Thus, it is important to understand dysfunctional reasoning biases within the separate traits of schizotypy with the different trait clusters likely having distinct mechanisms. No studies currently exist providing evidence of a significant relationship between the reasoning biases, JTC or BADE specifically, and the negative or disorganization traits of schizotypy. However, there is some evidence to suggest a relationship may exist between negative and disorganized schizotypy and JTC or BADE. For example, other studies have found evidence of a significant relationship between increased negative schizotypy traits and alternative reasoning biases such poorer theory of mind, greater attention for threat bias, and increased external attribution bias [9,31], each of whom are related to JTC and BADE [32]. Furthermore, negative schizotypy is characterized in part by lack of social, recreational, and vocational motivation which could reflect JTC and BADE deficits related to decreased anticipatory pleasure. Disorganized schizotypy in turn has been associated with context integration deficits, which potentially could signal a poor ability to consider or consolidate beliefs with updated contradictory evidence [33]. Perhaps a lack of published research in this area could signal that no relationship exists between negative or disorganized traits of schizotypy and these dysfunctional reasoning biases such as JTC or BADE, but this assertion stands to be tested.

The first aim of the current studies was to explore the relationships between perceived stress, schizotypy, and dysfunctional reasoning biases such as BADE and JTC. This was explored using two separate geographically-distinct samples of nonpsychiatric adults. We hypothesized that schizotypy and stress would significantly interact to predict dysfunctional reasoning biases such that elevated schizotypal traits and heightened perceived stress would predict increased reasoning biases. Our second aim was to examine whether biases were related to different trait clusters of schizotypy (i.e., positive, negative, and disorganized) as a function of varying levels of perceived stress. Based on the extant literature, we hypothesized that significant moderations would be observed for positive, but not negative or disorganized schizotypal traits.

\section{Methods}

\subsection{Participants}

Our sample included undergraduate students $(\mathrm{N}=236)$ recruited for separate studies at universities in the southeastern United States $(n=125)$ and northern England $(n=111)$. Demographic and clinical characteristics are included in Table 1 . This study was approved by the appropriate Human Subject Review Boards and subjects offered informed consent prior to completing the surveys.

\subsection{Schizotypy}

Schizotypy was measured using the Schizotypal Personality Questionnaire (SPQ) in both samples, although the English sample was administered the full version [34] and the US sample was administered the Brief-Revised version (SPQ-BR [35,36];). The full SPQ comprises 74 items with a true/false response format. The SPQ-BR was developed from the SPQ using both exploratory and confirmatory factor analysis to maximize internal consistency and factor independence. The response format of the SPQ-BR employs a five-point scale from "strongly disagree" to "strongly agree." Items $(n=32)$ reflect seven subordinate scales (i.e., odd/eccentric 
Table 1

Descriptive statistics for demographic and clinical variables for the two samples.

\begin{tabular}{|c|c|c|}
\hline Sex (\% Female) & $\frac{\text { US Sample (Study 1) }}{65 \%} n=125$ & $\frac{\text { English Sample (Study 2) }}{60 \%} n=111$ \\
\hline Age & $19.58 \pm 1.20$ & $22.14 \pm 4.79$ \\
\hline \multicolumn{3}{|l|}{ Schizotypal Traits ${ }^{\mathrm{a}}$} \\
\hline Cognitive-Perceptual & $31.86 \pm 10.39$ & $11.77 \pm 8.67$ \\
\hline Negative & $15.36 \pm 5.78$ & $11.63 \pm 7.17$ \\
\hline Disorganization & $24.35 \pm 7.41$ & $8.06 \pm 6.00$ \\
\hline Perceived Stress & $21.96 \pm 5.95$ & $15.87 \pm 6.35$ \\
\hline \multicolumn{3}{|l|}{ BADE Task ${ }^{\mathrm{b}}$} \\
\hline True response & $8.51 \pm 1.12$ & \\
\hline Emotional Lure response & $3.19 \pm 1.94$ & \\
\hline \multicolumn{3}{|l|}{ JTC (BEAD) Task } \\
\hline Trials & & $4.07 \pm 5.65$ \\
\hline Conclusion Accuracy & & $1.71 \pm 0.45$ \\
\hline
\end{tabular}

behavior, odd speech, constricted affect/no close friends, excessive social anxiety, unusual perceptual experiences, odd beliefs, ideas of reference/suspiciousness) and three superordinate factors (i.e., positive, negative, and disorganization; see [35,37].

\subsection{Stress}

Stress was assessed in both samples using the Perceived Stress Scale [22]. The PSS is comprised of 10 Likert-scale items $(0$ or $1=$ Never through to 4 or 5 = Very Often) of the frequency of perceived stress in the past month. The measure evaluated the perception of the unpredictability, uncontrollability, and overwhelming nature of an individual's life stress and was designed for community samples (e.g., In the last month . . . how often have you found that you could not cope with all the things that you had to do? [38];). The PSS has good psychometric properties with previous studies observing high internal consistency (Cronbach's $\alpha=0.85$ ) and is also correlated with self-reported physical health, health related behaviors, and help-seeking behaviors [39,40].

\subsection{Cognitive biases}

\subsection{1. $B A D E$}

Bias Against Disconfirmatory Evidence (BADE) was the cognitive bias measured in the US sample. To measure this, a previously used protocol [14] was employed that consisted of twelve written scenarios with four potential interpretations (i.e., "true," "emotional lure," "non-emotional lure," and "absurd") for each scenario. During three successive trials (i.e., sentences) for each scenario, additional progressively disambiguating information (i.e., disconfirmatory and confirmatory evidence) was introduced and the plausibility of each of the four interpretations were assessed by a slider scale ranging 1 10. Lure interpretations lose plausibility with additional information, whereas the true interpretation gains plausibility with additional information. The absurd interpretation was designed to appear implausible at all stages. An example of trials and interpretations for one written scenario [41] is provided here:

Trial 1: Jenny can't fall asleep

Trial 2: Jenny can't wait until it is finally morning.

Trial 3: Jenny wonders how many presents she will find under the tree.

Interpretation 1 (emotional lure): Jenny is worried about her ill mother.

Interpretation 2 (non-emotional lure): Jenny is nervous about her exam the next day.

Interpretation 3 (true): Jenny is excited about Christmas morning.
Interpretation 4 (absurd): Jenny loves her bed.

BADE should be reduced in light of inconsistent evidence within both lure conditions. More specifically, emotional lures have been previously observed to be a particularly sensitive measure of BADE in schizotypy $[14,41]$ and that reasoning biases should be considered within the framework that everyday decision making and everyday functioning occurs in contexts loaded with emotional valence [42]. The current study used the average of the emotional lure rating from the second and third trials as the measure of BADE consistent with prior studies [40], with increasing scores indicating higher BADE and therefore reasoning bias.

\subsubsection{JTC}

Jumping to conclusions (JTC) was assessed with the BEAD task in the English sample. In traditional versions of the BEAD task, there are only two jars present with opposing ratios of colored beads, which can lead to ceiling effects in performance for healthy volunteers due to its simplicity. Therefore, to increase the difficulty and uncertainty, three jars were presented at the same time to participants in the current study. The BEADS task consisted of two iterations of showing participants three jars of red and blue beads. The three jars were 85 red : 15 blue beads versus 50 red : 50 blue beads, versus 60 red : 40 blue bead ratios. The task was explained to participants with the jars on the screen and then the jars were removed from view. Participants were told that the experimenter would draw a series of beads one at a time (and without replacing them) from only one of the three jars. The beads were then shown one bead at a time to the participant, in a predetermined order. Participants were asked after they saw each bead whether they were ready to make a decision or whether they wanted to see an additional bead. Once participants indicated that they were ready to decide, they indicated which jar the beads were being drawn from. Jumping to a conclusion was defined as a decision based on very few beads seen. Consistent with prior studies $[43,44]$, the dependent variable (DV) were the number of beads seen (maximum of 20) before participants decided which of the three jar the beads were being drawn from and conclusion accuracy (binary outcome) for the 85:15 ratio condition, with fewer number of number of trials to conclusion (i.e., beads seen) and incorrect conclusion indicating greater JTC.

\subsection{Statistical analyses}

Analyses were conducted in two steps. First, data were inspected for normality and potential confounds. Second, interactions between an individual's schizotypal trait scores (i.e., total, positive, negative, and disorganized) and their perceived stress score were computed as predictors of BADE performance (US sample). These procedures (i.e., predictors and interactions) were 
Table 2

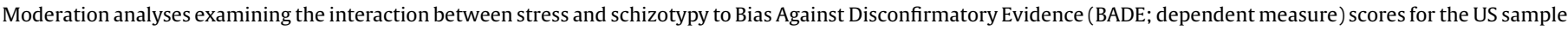
$(\mathrm{n}=125)$.

\begin{tabular}{|c|c|c|c|c|c|c|}
\hline \multicolumn{6}{|c|}{ BIAS AGAINST DISCONFIRMATORY EVIDENCE (EMOTIONAL LURES) } & \multirow[b]{2}{*}{$\underline{\mathrm{CI}}_{95 \mathrm{th} \%}$} \\
\hline & $\underline{\mathrm{R}}^{2}$ & $\underline{\mathrm{F}}$ & $\underline{\mathrm{B}}$ & $\underline{(\mathrm{SE})}$ & $\underline{\mathrm{t}}$ & \\
\hline Predictors & 0.00 & 0.17 & & & & \\
\hline Stress & & & 0.02 & 0.11 & 0.16 & -0.16 to 0.20 \\
\hline Positive Stypy & & & 0.11 & 0.11 & 0.97 & -0.08 to 0.29 \\
\hline Positive Stypy x Stress & 0.06 & $6.00^{*}$ & -0.22 & 0.09 & $-2.45^{*}$ & -0.36 to -0.07 \\
\hline Predictors & 0.01 & 0.48 & & & & \\
\hline Stress & & & 0.08 & 0.11 & 0.72 & -0.28 to 0.08 \\
\hline Negative Stypy & & & -0.10 & 0.11 & 0.94 & -0.20 to 0.15 \\
\hline Negative Stypy x Stress & 0.01 & 0.44 & 0.06 & 0.10 & 0.59 & -0.10 to 0.26 \\
\hline Predictors & 0.00 & 0.20 & & & & \\
\hline Stress & & & 0.06 & 0.11 & 0.59 & -0.12 to 0.24 \\
\hline Disorganized Stypy & & & -0.05 & 0.11 & -0.44 & -0.22 to 0.14 \\
\hline Disorg. Stypy x Stress & 0.03 & 0.95 & -0.14 & 0.09 & -1.57 & -0.29 to 0.01 \\
\hline
\end{tabular}

Note. Stypy = Schizotypy; Disorg. = Disorganized.

${ }^{*} p<0.05$.

Table 3

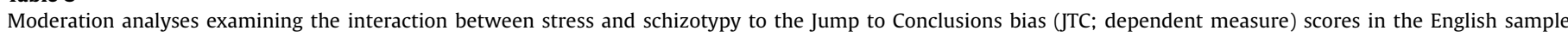
$(\mathrm{n}=111) . s$.

\begin{tabular}{|c|c|c|c|c|c|c|}
\hline \multicolumn{6}{|c|}{ JUMPING TO CONCLUSIONS BIAS (TRIALS) } & \\
\hline & $\mathrm{R}^{2}$ & $\mathrm{~F}$ & $\mathrm{~B}$ & $(\mathrm{SE})$ & $\mathrm{t}$ & \\
\hline Predictors & 0.01 & 0.48 & & & & \\
\hline Stress & & & -0.13 & 0.08 & 1.44 & -0.27 to 0.02 \\
\hline Positive Stypy & & & 0.04 & 0.09 & 0.46 & -0.11 to 0.19 \\
\hline Positive Stypy x Stress & 0.05 & 2.09 & 0.19 & 0.08 & $2.30^{*}$ & 0.05 to 0.32 \\
\hline Predictors & 0.01 & 0.52 & & & & \\
\hline Stress & & & -0.08 & 0.09 & -0.91 & -0.22 to 0.06 \\
\hline Negative Stypy & & & -0.07 & 0.09 & -0.84 & -0.25 to 0.03 \\
\hline Negative Stypy x Stress & 0.07 & $2.85^{*}$ & 0.21 & 0.07 & $2.73^{*}$ & 0.08 to 0.33 \\
\hline Predictors & 0.01 & 0.45 & & & & \\
\hline Stress & & & -0.13 & 0.09 & 1.42 & -0.28 to 0.02 \\
\hline Disorganized Stypy & & & 0.06 & 0.11 & 0.54 & -0.12 to 0.24 \\
\hline Disorg. Stypy x Stress & 0.04 & 1.49 & 0.20 & 0.11 & $1.88^{+}$ & 0.02 to 0.38 \\
\hline \multicolumn{7}{|c|}{ JUMPING TO CONCLUSIONS BIAS (CONCLUSION ACCURACY) } \\
\hline & $\mathrm{R}^{2}$ & $\mathrm{~F}$ & $\beta$ & $\mathrm{B}(\mathrm{SE})$ & $\mathrm{t}$ & $\mathrm{Cl}_{95 \mathrm{th} \%}$ \\
\hline Predictors & 0.01 & 0.60 & & & & \\
\hline Stress & & & 0.02 & 0.10 & 0.19 & 0.15 to 0.18 \\
\hline Positive Stypy & & & -0.11 & 0.11 & 1.05 & -0.17 to 0.15 \\
\hline Positive Stypy x Stress & 0.01 & 0.44 & 0.03 & 0.09 & 0.33 & -0.13 to 0.19 \\
\hline Predictors & 0.01 & 0.67 & & & & \\
\hline Stress & & & 0.02 & 0.10 & 0.23 & -0.14 to 0.19 \\
\hline Negative Stypy & & & -0.14 & 0.10 & 1.43 & -0.31 to 0.02 \\
\hline Negative Stypy x Stress & 0.04 & 1.44 & 0.15 & 0.09 & $1.72+$ & 0.00 to 0.29 \\
\hline Predictors & 0.03 & 2.02 & & & & \\
\hline Stress & & & 0.07 & 0.10 & 0.65 & -0.10 to 0.24 \\
\hline Disorganized Stypy & & & -0.24 & 0.13 & $1.93+$ & -0.44 to -0.03 \\
\hline Disorg. Stypy x Stress & 0.03 & 1.34 & 0.00 & 0.12 & 0.01 & -0.20 to 0.20 \\
\hline
\end{tabular}

repeated for JTC performance [e.g., number of trials (beads seen), conclusion accuracyl as the criterion variable (English Sample). See Table 2 and 3 for all permutations of linear regression models for the three criterion variables (e.g., BADE performance, JTC - number of trials, and JTC - conclusion accuracy). Analyses were conducted in R. Significant interactions were evaluated using simple slopes analysis. Unless otherwise noted, all variables were normally distributed. Standardized scores for the criterion and predictors variables were used in all figures.

\section{Results}

\subsection{Preliminary analyses}

Relationships between the JTC/BADE measures and demographic variables (i.e., gender, ethnicity, and age) were examined to evaluate potential demographic confounds. No significant results of note emerged.

\subsection{BADE analyses - US sample study}

Results for the BADE analyses in the US sample study are presented in Table 2. Schizotypy and perceived stress scores were not independently associated with BADE performance; this was pattern observed for all schizotypy traits (i.e., positive, negative, and disorganized). However, there was a significant interaction between positive schizotypy and stress $[\mathrm{B}(\mathrm{SE})=-.22(.09), \mathrm{p}<.05]$. Simple slopes analysis suggested that increased stress was associated with increased BADE (i.e., greater reasoning bias) in individuals with low positive schizotypy at trend level $[\mathrm{B}(\mathrm{SE})=.23$ $(.14), p<.10)$. In individuals with high positive schizotypal scores, stress was not related BADE performance $[\mathrm{B}(\mathrm{SE})=-.20(.14), p=.17]$. 


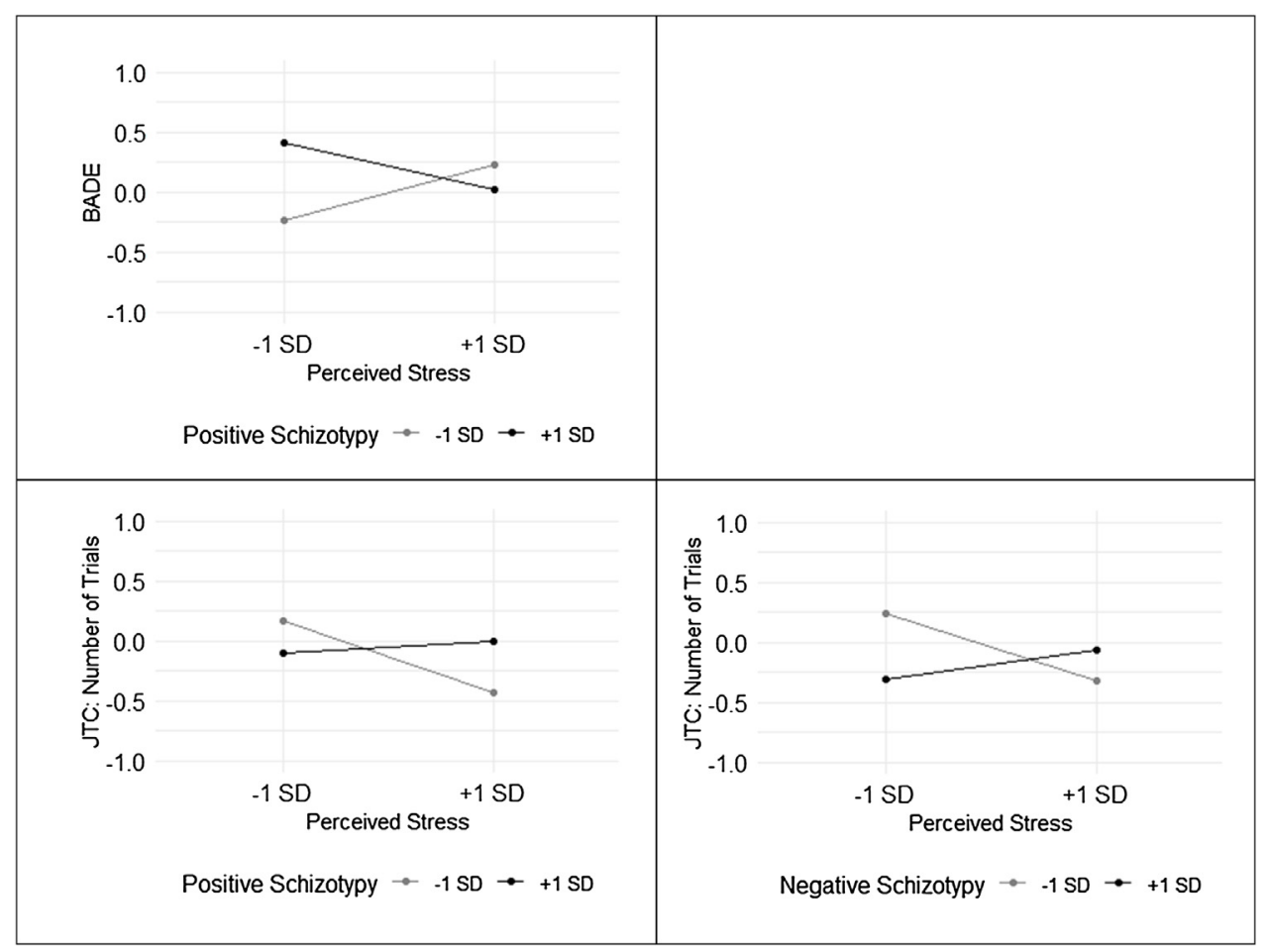

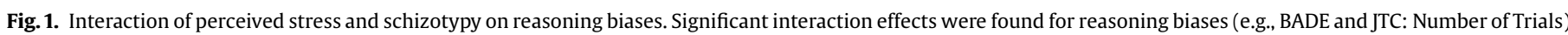

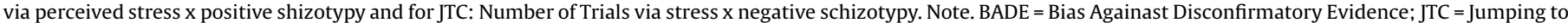
Conclusions; Gray line $=1$ SD below (Low Schizotypy); Black line $=1$ SD above (High Schizotypy). All criterion and predictor variables are standardized.

Graphic illustration of simple slopes (see Fig. 1) indicate that individuals with positive schizotypy showed high levels of BADE at low levels of stress thus suggesting that their BADE tended to be relatively high and stress-resistant.

\subsection{JTC analyses - English sample study}

Results for JTC analyses in the English sample study are presented in Table 3. Neither schizotypy traits (i.e., positive, negative, and disorganized) nor stress were significantly associated with JTC performance independently. However, significant interactions between only positive $[\mathrm{B}(\mathrm{SE})=.19(.08), \mathrm{p}<.05]$ and negative schizotypy traits $[\mathrm{B}(\mathrm{SE})=.21(.07), \mathrm{p}<.05]$ and stress were observed for predicting the number of trials to reach a conclusion. No significant interaction existed between disorganized schizotypy traits and perceived stress. Simple slopes analysis suggested that increased stress was associated with fewer number of trials needed to reach conclusion (i.e., reflecting a faster JTC) in individuals with low positive schizotypy $[\mathrm{B}(\mathrm{SE})=-.30(.13)$, $\mathrm{p}<.05]$. However, in individuals with high positive schizotypal scores, stress was not related JTC performance $[\mathrm{B}(\mathrm{SE})=.05(.10)$, $\mathrm{p}=.65$ ]. Graphic illustrations of simple slopes (see Fig. 1) indicate that individuals with positive schizotypy showed higher levels of JTC (i.e., lower task performance via fewer number of trials needed to reach conclusion) even at low levels of stress. This suggested that their JTC tendencies were relatively high and stress-resistant. Unexpectedly, a significant interaction between negative schizotypy and stress was also observed (see Fig. 1). The pattern of results was largely similar to that seen with positive schizotypy. Individuals low in negative schizotypal traits showed decreased task performance as a function of increased perceived stress [B(SE) $=-.28(.12), \mathrm{p}<.05]$. Graphic illustrations of simple slopes (see Fig. 1) indicate that individuals with negative schizotypy showed abnormally high levels of JTC (i.e., lower task performance via fewer number of trials needed to reach conclusion) even at low levels of stress. Similar to the BADE results, individuals with elevated positive and negative schizotypy exhibited task performance (via greater number of trials completed) that was largely immutable to stress, contrary to hypotheses. The interaction term for stress and schizotypy factors did not account for significant variance in JTC performance via conclusion accuracy.

\section{Discussion}

These studies evaluated how dysfunctional reasoning biases and schizotypy traits (i.e., positive, negative, and disorganized) varied as a function of perceived stress. Schizotypy traits were not, in and of themselves, related to reasoning biases in either of the samples, which is consistent with prior studies that show schizotypy is largely associated with normal performance on objective task cognitive tasks [45]. When accounting for stress, however, significant relationships were observed. Reasoning biases were relatively dynamic as a function of stress in individuals with overall low schizotypal traits. In individuals with high levels of positive schizotypal traits, these reasoning biases appeared pronounced at relatively low stress levels and appeared to be less dynamic as a function of stress, though as noted earlier, schizotypal traits were not independently linked to reasoning biases. Similar patterns were observed for negative schizotypy with respect to the JTC bias (i.e. English sample), but not BADE (i.e., US sample). Significant relationships between reasoning biases and disorganized schizotypy were not observed in either sample lending support for specificity. Given that BADE and JTC both tap into decision making under conditions of uncertainty, results from these studies have ecological validity for the conditions encountered in everyday life. Indeed, it is important to understand the 
effects of perceived stress on these reasoning biases considering that much of decision making within everyday functioning occurs in contexts loaded with emotional valence.

The finding that reasoning biases appeared to emerge in individuals with positive schizotypal traits during a period (i.e., past month) of low stress is fairly unsurprising as these biases are included in etiological theories. There were several surprising findings however. First, we found that reasoning biases in positive schizotypy were relatively "stress-resistant", that is, their reasoning biases were relatively consistent regardless of perceived stress levels. This did not appear to reflect "ceiling effects" from any of the measures used in this study as care was taken to ensure that both tasks were sufficiently challenging for healthy volunteers to ensure 'perfect' performance could not be achieved. Second, main effects between the schizotypy factors and reasoning biases measures (BADE and JTC) were not observed and, generally speaking, significant interactions were in the small effect size range. Our sample consisted of relatively high functioning individuals on the psychosis continuum as we assessed schizotypal traits dimensionally rather than using an enriched, schizotypy sample (i.e., higher likelihood of clinical symptomatology). However, this makes it even more vital to consider interactions with relevant moderators such as perceived psychological stress in a nonclinical sample. Our findings (i.e., significant interaction) likely reflects an underestimate of true effects. In this case, our findings would likely generalize to individuals "further along" on the psychosis continuum and main effects and significant interactions with medium to large effects would likely be observed. Finally, consistent findings across both of our studies using different measures engenders confidence in our findings and not merely spurious.

Because these results may have mechanistic implications, some cautious neurobiological interpretations of these results are warranted. Prefrontal-limbic connections are important in reasoning biases, aberrant salience, acute and chronic stress [46], and in key dopamine pathways theorized to be affected in individuals experiencing psychotic symptoms and/or schizophrenia spectrum traits more broadly $[46,47]$. The prefrontal cortex functions optimally with a narrow range of dopamine levels, but factors such as stress have been shown to affect dopamine levels [29]. This may explain why individuals low in schizotypal traits, who likely have intact prefrontal pathways and structures, appeared to exhibit JTC only at high levels of stress. If an individual has less than optimal prefrontal and dopamine functioning, such as those who have elevated schizotypal traits [48], then stress may have less of an impact. Due to the cross-sectional nature of our studies, future research would benefit by examining reasoning biases following experimentally or ecologically induced stress while also recording physiological reactivity and dopaminergic functioning (i.e., eyeblink rate as proxy). Indeed, several previous studies have examined associations between state negative affect, dysfunctional reasoning biases, and delusional thought processes in daily life within the psychosis spectrum. Collectively, these studies found that laboratory and ecologically measured JTC were linked with state delusional experiences [49,50], though not necessarily state negative such as stress [51].

One other important interpretation of these results is seeing these cognitive biases as beneficial strategies of reasoning in some contexts. Given that JTC biases are associated with quicker decision times and not always less accurate decisions, this and BADE may be evolutionarily adaptive. Indeed, schizotypy traits, and their interactions with perceived stress, were not associated with performance accuracy on our JTC task. From a survival perspective, quicker decisions are advantageous, especially in the presence of threat when speed is essential. Perhaps individuals low in schizotypal traits experiencing higher perceived stress revert to a more efficient decision-making strategy as threat and perceived stress increases. Thus, increased cognitive biases may be adaptive in the short-term and not necessarily dysfunctional to an individual's daily life. With that said, the current studies examined perceived stress in a relatively larger temporal epoch (i.e., past month) rather than an acute, short-term stressor. As noted earlier, misinterpretation regarding threat is common in those with schizophrenia-spectrum disorders and these misinterpretations may be present notwithstanding the severity of perceived stress within the past month as our findings suggest. These dysfunctional cognitive biases represent vital treatment targets across multiple widely implemented interventions [17]. Further research on the potentially adaptive utility of cognitive biases in daily life in clinical and nonclinical populations is warranted.

There are several limitations of these current studies worth noting. First, subjects were drawn from undergraduate populations, so they likely reflect some of the highest functioning individuals on the psychosis continuum. This sample's neurocognitive functioning, while potentially less than optimal in individuals high in schizotypal traits, far surpasses that of individuals with schizophrenia [25]. It is unclear to what degree results from these studies generalize to individuals with more severe functional deficits from schizotypy. This sample may have a higher level of functioning because of adaptive beliefs, strategies, and other resilience factors - which could include effective stress management techniques. Hence, the present studies may highlight resources that help mitigate potentiallydysfunctional effects of stress on cognitive systems. That being said, reasoning biases in schizotypy appeared to be pronounced even at low-stress levels suggesting dysfunctional cognitive biases are occurring even in the context of low perceived threat situations. For individuals with elevated schizotypal traits, low levels of stress may have similar effects on reasoning systems as those observed in other individuals only under high levels of stress and our current results support this interpretation. The lack of change in reasoning biases associated with schizotypal traits could reflect a near asymptotic performance (achieved under much lower levels of stress compared to individuals with low schizotypy). Secondly, our understanding of perceived stress was limited to self-report, and our methodology was correlational in design. Future research could experimentally manipulate acute stress levels to evaluate their direct effects on reasoning biases. Stress levels could also be confirmed using physiological measures, although as noted previously, the subjective experience of stress in individuals with schizotypy may be more meaningful to examine. Relatedly, a meta-analysis by Ross and colleagues [19] highlighted the current studies' limitations with the BEAD task. Namely, that associations between the JTC obtained via the BEAD task and delusion formation and maintenance varied in effect size due to confounds such as current psychiatric symptoms, negative symptoms, IQ, working memory capabilities, and sample size. Moreover, the BEAD task distributed beads in a fixed order which may lead to order effects. Finally, the BADE task itself has been subject to scrutiny regarding varying scoring methodology [52] and this represents a limitation in our studies as well (i.e., exclusively using emotional lures). Future research should attempt to extend our findings by using alternative laboratory-based reasoning biases tasks.

These limitations notwithstanding, the present studies provided some of the first consistent evidence that reasoning biases are linked with perceived stress, yet only with respect to positive schizotypy traits. This finding has important implications for intervention and perhaps even predictive models of positive symptoms. If individuals high in positive schizotypy traits exhibit reasoning biases regardless of perceived level of stress, using traitlike measures of perceived stress may be sufficient and other 
dynamic factors can be explored as vulnerability factors. In light of calls for replicability, future studies can use mobile assessments to explore the influence of acute stress and other dynamic factors (e.g., loneliness, impulsivity, physiological arousal) on reasoning biases in daily life. Further understanding about the role of stress and dopamine as they relate to psychotic symptoms can enhance our treatments, specifically pharmacological treatments already targeting this system. Much is left to be explored, but these studies delved deeper into the sequelae, and potentially specific mechanism, of reasoning biases in individuals experiencing symptoms on the schizophrenia spectrum.

\section{Role of source funding}

None.

\section{Contributors}

$\mathrm{TL}, \mathrm{TF}$, and $\mathrm{AC}$ performed the literature search and wrote the majority of the manuscript. TL, AC, and EB conducted statistical analysis and data interpretation. SA and $\mathrm{CP}$ also assisted with the literature search. AC, EB, and SL designed the parent studies. All authors contributed to the writing of the manuscript.

\section{Declaration of Competing Interest}

The authors report no conflict of interest.

\section{Acknowledgements}

The authors would like to thank the study participants and lab members for their aid in collecting and processing data.

\section{References}

[1] Chong HY, Teoh SL, DB-C Wu, Kotirum S, Chiou C-F, Chaiyakunapruk N. Global economic burden of schizophrenia: a systematic review. Neuropsychiatr Dis Treat 2016;12:357.

[2] Knapp M, Mangalore R, Simon J. The global costs of schizophrenia. Schizophr Bull 2004;30:279.

[3] Conus P, Cotton S, Schimmelmann BG, McGorry PD, Lambert M. The FirstEpisode Psychosis Outcome Study: premorbid and baseline characteristics of an epidemiological cohort of 661 first-episode psychosis patients. Early Interv Psychiatry 2007;1:191-200, doi:http://dx.doi.org/10.1111/j.17517893.2007.00026.x.

[4] Lenzenweger MF. Schizotypy: An Organizing Framework for Schizophrenia Research. Curr Dir Psychol Sci 2006;15:162-6, doi:http://dx.doi.org/10.1111/ j.1467-8721.2006.00428.x.

[5] Kwapil TR, Barrantes-Vidal N. Schizotypy: Looking Back and Moving Forward. Schizophr Bull 2014;41:S366-73, doi:http://dx.doi.org/10.1093/ schbul/sbu186.

[6] Vollema M, Sitskoorn M, Appels M, Kahn R. Does the Schizotypal Personality Questionnaire reflect the biological-genetic vulnerability to schizophrenia? Schizophr Res 2002;54:39-45, doi:http://dx.doi.org/10.1016/S0920-9964(01) 00350-4.

[7] Docherty AR, Fonseca-Pedrero E, Debbané M, Chan RCK, Linscott RJ, et al. Enhancing psychosis-spectrum nosology through an international data sharing initiative. Schizophr Bull 2018, doi:http://dx.doi.org/10.1093/schbul/sby059.

[8] Stefanis NC, Trikalinos TA, Avramopoulos D, Smyrnis N, Evdokimidis I, Ntzani $\mathrm{EE}$, et al. Impact of schizophrenia candidate genes on schizotypy and cognitive endophenotypes at the population level. Biol Psychiatry 2007;62:784-92, doi: http://dx.doi.org/10.1016/J.BIOPSYCH.2006.11.015.

[9] Sacks SA, Weisman de Mamani AG, Garcia CP. Associations between cognitive biases and domains of schizotypy in a non-clinical sample. Psychiatry Res 2012;196:115-22, doi:http://dx.doi.org/10.1016/J.PSYCHRES.2011.09.019.

[10] Snitz BE, MacDonald AW, Carter CS. Cognitive deficits in unaffected firstdegree relatives of schizophrenia patients: A meta-analytic review of putative endophenotypes. Schizophr Bull 2005;32:179-94, doi:http://dx.doi.org/ $10.1093 / \mathrm{schbul} / \mathrm{sbi048}$

[11] McLean BF, Mattiske JK, Balzan RP. Association of the jumping to conclusions and evidence integration biases with delusions in psychosis: a detailed meta-analysis. Schizophr Bull 2017, doi:http://dx.doi.org/10.1093/ schbul/sbw056.
[12] Pinkham AE, Harvey PD, Penn DL. Paranoid individuals with schizophrenia show greater social cognitive bias and worse social functioning than nonparanoid individuals with schizophrenia. Schizophr Res Cogn 2016, doi:http:// dx.doi.org/10.1016/j.scog.2015.11.002.

[13] Juarez-Ramos V, Rubio J, Delpero C, Mioni G, Stablum F, Gomez-Milan E. Jumping to conclusions bias, BADE and feedback sensitivity in schizophrenia and schizotypy. Conscious Cogn 2014;26:133-44.

[14] Woodward TS, Buchy L, Moritz S, Liotti M. A bias against disconfirmatory evidence is associated with delusion proneness in a nonclinical sample. Schizophr Bull 2007;33:1023-8.

[15] Garety PA, Freeman D. Cognitive approaches to delusions: a critical review of theories and evidence. Br J Clin Psychol 1999;38:113-54.

[16] Garety P, Joyce E, Jolley S, Emsley R, Waller H, Kuipers E, et al. Neuropsychological functioning and jumping to conclusions in delusions. Schizophr Res 2013;150:570-4, doi:http://dx.doi.org/10.1016/J.SCHRES.2013.08.035.

[17] Penn DL, Roberts DL, Combs D, Sterne A. Best practices: the development of the Social Cognition and Interaction Training Program for schizophrenia spectrum disorders. Psychiatr Serv 2007;58:449-51, doi:http://dx.doi.org/10.1176/ ps.2007.58.4.449.

[18] Moritz S, Woodward TS. Metacognitive training in schizophrenia: from basic research to knowledge translation and intervention. Curr Opin Psychiatry 2007;20:619-25.

[19] Ross RM, McKay R, Coltheart M, Langdon R. Jumping to conclusions about the beads task? A meta-analysis of delusional ideation and data-gathering. Schizophr Bull 2015;41:1183-91.

[20] Tsakanikos E. Perceptual biases and positive schizotypy: The role of perceptual load. Pers Individ Dif 2006;41:951-8.

[21] Lincoln TM, Lange J, Burau J, Exner C, Moritz S. The effect of state anxiety on paranoid ideation and jumping to conclusions. An experimental investigation. Schizophr Bull 2010;36:1140-8, doi:http://dx.doi.org/10.1093/schbul/sbp029.

[22] Cohen S, Kamarck T, Mermelstein R. A global measure of perceived stress. J Health Soc Behav 1983;24:385-96.

[23] Keefe KM, Warman DM. Reasoning, delusion proneness and stress: an experimental investigation. Clin Psychol Psychother 2011, doi:http://dx.doi. org/10.1002/cpp.683.

[24] Medlin H, Warman D. An investigation into reasoning biases, mood and cognitive state, and subclinical delusional ideation. Psychiatry Res 2014, doi: http://dx.doi.org/10.1016/j.psychres.2014.07.014.

[25] Cohen AS, Auster TL, MacAulay RK, McGovern JE. The paradox of schizotypy: resemblance to prolonged severe mental illness in subjective but not objective quality of life. Psychiatry Res 2014;217:185-90, doi:http://dx.doi.org/10.1016/j. psychres.2014.03.016.

[26] Li LY, Karcher NR, Kerns JG, Fung CK, Martin EA. The subjective-objective deficit paradox in schizotypy extends to emotion regulation and awareness. J Psychiatr Res 2019, doi:http://dx.doi.org/10.1016/j.jpsychires.2019.01.026.

[27] Suhr J, Spitznagel M. Factor versus cluster models of schizotypal traits. I: a comparison of unselected and highly schizotypal samples. Schizophr Res 2001;52:231-9, doi:http://dx.doi.org/10.1016/S0920-9964(00)00170-5.

[28] Li Y, Hou X, Wei D, Du X, Zhang Q, Liu G, et al. Long-term effects of acute stress on the prefrontal-limbic system in the healthy adult. PLoS One 2017, doi:http:// dx.doi.org/10.1371/journal.pone.0168315.

[29] Howes OD, McCutcheon R, Owen MJ, Murray RM. The role of genes, stress, and dopamine in the development of schizophrenia. Biol Psychiatry 2017, doi: http://dx.doi.org/10.1016/j.biopsych.2016.07.014

[30] Menon M, Quilty LC, Zawadzki JA, Woodward TS, Sokolowski HM, Boon HS et al. The role of cognitive biases and personality variables in subclinical delusional ideation. Cogn Neuropsychiatry 2013;18:208-18, doi:http://dx.doi. org/10.1080/13546805.2012.692873.

[31] Gawęda Ł, Prochwicz K, Cella M. Cognitive biases mediate the relationship between temperament and character and psychotic-like experiences in healthy adults. Psychiatry Res 2015;225:50-7, doi:http://dx.doi.org/10.1016/j. psychres.2014.10.006.

[32] Mizrahi R. Social stress and psychosis risk: common neurochemica substrates? Neuropsychopharmacology 2016, doi:http://dx.doi.org/10.1038/ npp.2015.274.

[33] Chun CA, Ciceron L, Kwapil TR. A meta-analysis of context integration deficits across the schizotypy spectrum using AX-CPT and DPX tasks. J Abnorm Psychol 2018, doi:http://dx.doi.org/10.1037/abn0000383.

[34] Raine A. The SPQ: A scale for the assessment of schizotypal personality based on DSM-III-R criteria. Schizophr Bull 1991;17:555-64.

[35] Callaway DA, Cohen AS, Matthews RA, Dinzeo T. Schizotypal personality questionnaire-brief revised: psychometric replication and extension. Personal Disord 2014;5:32-8, doi:http://dx.doi.org/10.1037/per0000041.

[36] Cohen AS, Matthews RA, Najolia GM, Brown LA. Toward a more psychometrically sound brief measure of schizotypal traits: introducing the SPQ-Brief Revised. J Pers Disord 2010;24:516-37, doi:http://dx.doi.org/ 10.1521/pedi.2010.24.4.516.

[37] Fonseca-Pedrero E, Chan RCK, Debbané M, Cicero D, Zhang LC, et al Comparisons of schizotypal traits across 12 countries: Results from the International Consortium for Schizotypy Research. Schizophr Res 2018, doi: http://dx.doi.org/10.1016/j.schres.2018.03.021.

[38] Cohen S, Williamson G. Perceived stress in a probability sample of the United States. In: Spacapan S, Oskamp S, editors. Soc. Psychol. Heal.. Newbury Park, CA: Sage; 1988. p. 31-67. 
[39] Teh HC, Archer JA, Chang W, Chen SHA. Mental well-being mediates the relationship between perceived stress and perceived health. Stress Health 2015, doi:http://dx.doi.org/10.1002/smi.2510.

[40] Vankim NA, Nelson TF. Vigorous physical activity, mental health, perceived stress, and socializing among college students. Am J Health Promot 2013, doi: http://dx.doi.org/10.4278/ajhp.111101-QUAN-395.

[41] Buchy L, Woodward TS, Liotti M. A cognitive bias against disconfirmatory evidence (BADE) is associated with schizotypy. Schizophr Res 2007, doi:http:/ dx.doi.org/10.1016/j.schres.2006.11.012.

[42] Coan JA, Allen JJB, McKnight PE. A capability model of individual differences in frontal EEG asymmetry. Biol Psychol 2006, doi:http://dx.doi.org/10.1016/j. biopsycho.2005.10.003.

[43] Dudley R, Taylor P, Wickham S, Hutton P. Psychosis, delusions and the "jumping to conclusions" reasoning bias: a systematic review and metaanalysis. Schizophr Bull 2015;42:652-65.

[44] Van Dael F, Versmissen D, Janssen I, Myin-Germeys I, et al. Data gathering: biased in psychosis? Schizophr Bull 2006;32:341-51, doi:http://dx.doi.org/ 10.1093/schbul/sbj021.

[45] Chun CA, Minor KS, Cohen AS. Neurocognition in psychometrically defined college Schizotypy samples: we are not measuring the "right stuff". J Int Neuropsychol Soc 2013;19:324-37, doi:http://dx.doi.org/10.1017/ S135561771200152X

[46] Pani L, Porcella A, Gessa G. The role of stress in the pathophysiology of the dopaminergic system. Mol Psychiatry 2000;5:14.
[47] Broyd A, Balzan RP, Woodward TS, Allen P. Dopamine, cognitive biases and assessment of certainty: a neurocognitive model of delusions. Clin Psychol Rev 2017;54:96-106.

[48] Rössler J, Unterassner L, Wyss $T$, Haker H, Brugger P, Rössler W, et al Schizotypal Traits are Linked to Dopamine-Induced Striato-Cortical Decoupling: A Randomized Double-Blind Placebo-Controlled Study. Schizophr Bull 2018, doi:http://dx.doi.org/10.1093/schbul/sby079.

[49] Ben-Zeev D, Morris S, Swendsen J, Granholm E. Predicting the occurrence, conviction, distress, and disruption of different delusional experiences in the daily life of people with schizophrenia. Schizophr Bull 2012;38:826-37, doi: http://dx.doi.org/10.1093/schbul/sbq167.

[50] wai So SH, Peters ER, Swendsen J, Garety PA, Kapur S. Changes in delusions in the early phase of antipsychotic treatment - an experience sampling study. Psychiatry Res 2014, doi:http://dx.doi.org/10.1016/j.psychres.2013.12.033.

[51] Lüdtke T, Kriston L, Schröder J, Lincoln TM, Moritz S. Negative affect and a fluctuating jumping to conclusions bias predict subsequent paranoia in daily life: an online experience sampling study. J Behav Ther Exp Psychiatry 2017, doi:http://dx.doi.org/10.1016/j.jbtep.2016.08.014.

[52] Bronstein MV, Cannon TD. Measuring bias against disconfirmatory evidence: An evaluation of BADE task scoring methods and the case for a novel method. Psychiatry Res 2018, doi:http://dx.doi.org/10.1016/j. psychres.2018.01.007. 